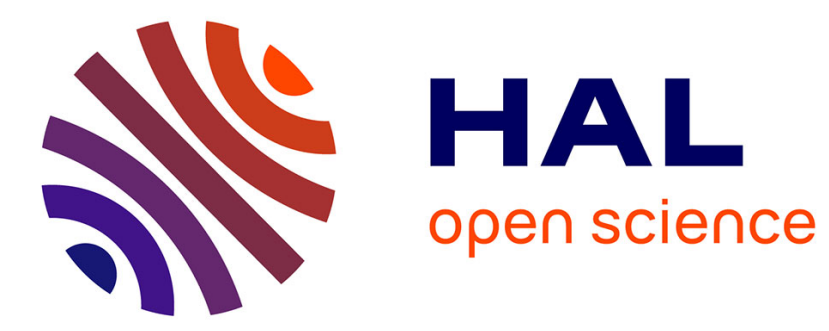

\title{
Jonctions à localisation associées à un spectromètre Buechner
}

\author{
G. Lemarchand, J. Quidort
}

\section{To cite this version:}

G. Lemarchand, J. Quidort. Jonctions à localisation associées à un spectromètre Buechner. Revue de Physique Appliquée, 1969, 4 (2), pp.105-106. 10.1051/rphysap:0196900402010500 . jpa-00243165

\section{HAL Id: jpa-00243165 https://hal.science/jpa-00243165}

Submitted on 1 Jan 1969

HAL is a multi-disciplinary open access archive for the deposit and dissemination of scientific research documents, whether they are published or not. The documents may come from teaching and research institutions in France or abroad, or from public or private research centers.
L'archive ouverte pluridisciplinaire HAL, est destinée au dépôt et à la diffusion de documents scientifiques de niveau recherche, publiés ou non, émanant des établissements d'enseignement et de recherche français ou étrangers, des laboratoires publics ou privés. 


\title{
JONGTIONS A LOGALISATION ASSOGIÉES A UN SPEGTROMÈTRE BUEGHNER
}

\author{
G. LEMARCHAND et J. QUIDORT, \\ Service de Physique Nucléaire à Basse Énergie, C.E.N., Saclay.
}

\begin{abstract}
Résumé. - Nous décrivons un système détecteur de particules installé sur le spectromètre magnétique Buechner du $\mathrm{D}$. Ph-N/BE. Il comporte quatre jonctions à localisation de $50 \mathrm{~mm} \times 15 \mathrm{~mm}$ suivies d'un diviseur analogique. La résolution spatiale atteint $0,7 \mathrm{~mm}$ et la non-linéarité de réponse est inférieure à $5 \%$.
\end{abstract}

Abstract. - We describe a particle detector system set-up for the Saclay Buechner spectrometer. It includes four position sensitive detectors (active area $50 \times 15 \mathrm{~mm}$ ) connected to pulse voltage dividing circuit. The space resolution is $0.7 \mathrm{~mm}$ and the non-linearity position is less than $5 \%$.

Le spectromètre Buechner nécessite un système de détection placé sur sa courbe focale qui indique le lieu d'impact des particules incidentes. Les émulsions ionographiques utilisées actuellement ne permettent pas les mesures en temps réel, quelle que soit la méthode de dépouillement utilisée : observateur humain ou système automatique lecteur de traces.

La jonction à localisation nous a paru être le détecteur souhaitable pour plusieurs raisons : 1) mesure en temps réel; 2) résolution spatiale de $0,5 \mathrm{~mm}$ pour une longueur de $50 \mathrm{~mm}$, donc compatible avec notre problème; 3) contrairement aux chambres à étincelles classiques, la fenêtre d'entrée est actuellement mince, puisqu'elle se réduit à une barrière de surface; il sera éventuellement possible de localiser des particules lourdes de basse énergie; 4) l'angle d'incidence des particules sur le détecteur n'influence pas sa résolution spatiale du fait qu'une information élémentaire est constituée par un nombre important de paires électronstrous créées par la trajectoire de la particule dans la zone désertée; 5) dans la plupart des cas, ces détecteurs pourront nous renseigner sur la masse des particules.

Ce détecteur délivrant une information $E$ relative à l'énergie perdue par la particule et une information $E \mathrm{f}(x)$ relative à cette énergie et au lieu d'impact, il se révèle indispensable d'effectuer un quotient si l'on veut bénéficier d'un étalonnage en position indépendant de l'énergie. Deux méthodes semblent possibles pour effectuer ce quotient : soit l'emploi d'un calculateur analogique en temps réel, soit l'emploi d'un calculateur numérique en ligne. Le calculateur analogique nous a séduits par sa relative simplicité et son prix de revient peu élevé. Toutefois, une réalisation soignée est nécessaire pour obtenir les performances désirables.
Quelques auteurs, en particulier Tsukuda [1] et Broomall [2], ont décrit des diviseurs analogiques. Le circuit réalisé ici utilise la montée linéaire de tension, en fonction du temps, aux bornes d'une capacité chargée à courant constant. Soient $C_{1}$ et $C_{2}$ les capacités relatives aux voies $E$ et $E f(x), V_{1}$ et $V_{2}$ les tensions correspondantes. On montre que la tension donnée par le quotient est $V=\left(V_{2} / V_{1}\right) \times V_{\mathrm{s}}$, où $V_{\mathrm{s}}$ est une tension fixe de seuil d'échantillonnage. La durée des signaux de charge des capacités a été fixée à $2 \mu$ s et celle de l'échantillonnage à $200 \mathrm{~ns}$, la précision du quotient obtenu atteint $0,5 \%$ dans une dynamique de $24 \mathrm{~dB}$.

Les quatre détecteurs employés sont des « Nuclear Diodes NTC 1450-450 ». Un circuit de codage et un mélangeur précédé de portes linéaires permettent de n'utiliser qu'un circuit diviseur et un seul convertisseur analogique digital pour ces quatre détecteurs.

Un premier essai a consisté à placer, devant une jonction à localisation, une source de ${ }^{241} \mathrm{Am}$ avec interposition d'un écran percé de fenêtres rectangulaires de $1 \mathrm{~mm}$ de large, espacées de $2 \mathrm{~mm}$, afin de définir des zones où pénètrent les particules $\alpha$ de $5,4 \mathrm{MeV}$ dans le détecteur. La réponse du quotient est proche d'une droite; l'erreur maximale atteint $5 \%$. Avec le spectromètre Buechner, nous avons vérifié le bon fonctionnement du dispositif pour les particules de $6,05 \mathrm{MeV}$ de ${ }^{212} \mathrm{Bi}$ et $8,78 \mathrm{MeV}$ de ${ }^{212} \mathrm{Po}$ (fig. 1). Le léger écart entre ces deux courbes peut provenir d'une erreur systématique sur la valeur du champ magnétique, puisqu'elles sont parallèles; il est néanmoins possible de constater qu'avec le quotient le système est sensible uniquement à la position $\mathrm{f}(x)$. On a pu séparer les particules $\alpha$ de $6,0497 \mathrm{MeV}$ et $6,0898 \mathrm{MeV}$ $\mathrm{de}^{212} \mathrm{Bi}$. La résolution spatiale est $0,7 \mathrm{~mm}$ (soit $5,1 \mathrm{keV}$ ), 


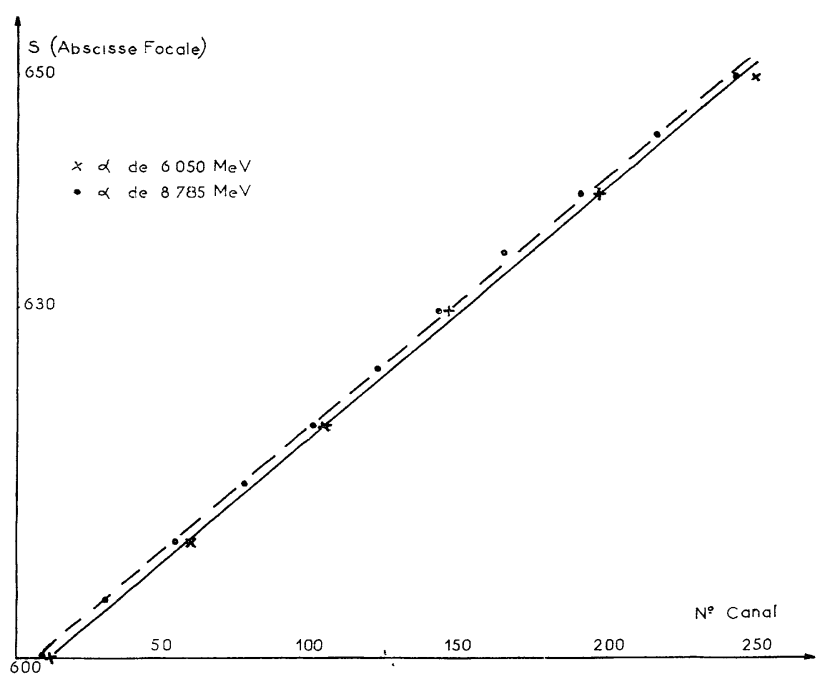

FIG. 1.

résultat comparable à ceux obtenus avec les émulsions nucléaires. L'angle d'incidence $25^{\circ}$ des particules sur le détecteur ne détériore pas la résolution du système.

Les premiers essais avec une réaction ${ }^{27} \mathrm{Al}(\mathrm{d}, \mathrm{p})^{28} \mathrm{Al}$ nous ont montré : 1) qu'il était possible de discriminer les protons des deutons inélastiques (d'énergie moitié), arrivant au même endroit sur le détecteur; 2) que la résolution avec des cibles minces atteignait $9,5 \mathrm{keV}$ pour des protons de $17, \overline{\mathrm{J}} \mathrm{MeV}$, soit $0, \overline{\mathrm{a}} 4 \%$, ce qui correspond pratiquement à la résolution intrinsèque du spectrographe (fig. 2).

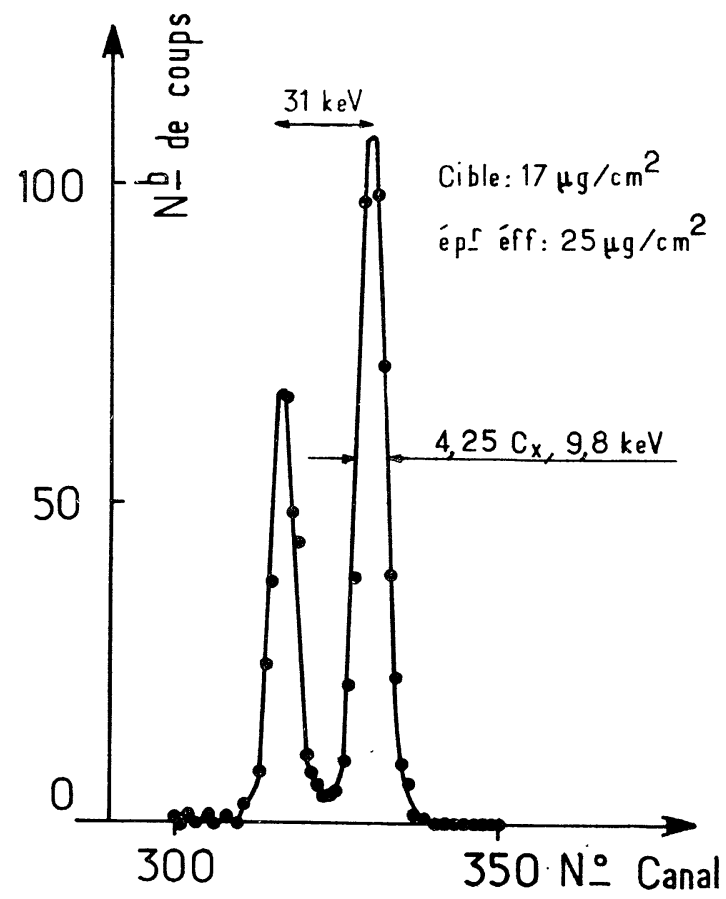

FIG. 2. $-{ }^{27} \mathrm{Al}(\mathrm{d}, \mathrm{p})^{28} \mathrm{Al}, E_{\mathrm{d}}=12 \mathrm{MeV}$.

\section{BIBLIOGRAPHIE}

[1] Tsukuda (M.), Nucl. Instr. Methods, 1964, 25, 265.

[2] Broomali (J.) et Riebman, Proc. I.R.E., 1952, 40, 568 . 